M. TAKANO

KODAI MATH. J.

12 (1989), 125-131

\title{
INTERPOLATION THEOREM IN MANY-VALUED LOGICS WITH DESIGNATED VALUES
}

\author{
Dedicated to Professor S. Maehara on his 60th birthday
}

By Mitio TAKano

\section{§ 0. Introduction.}

Many-valued logics can be divided into two categories according as the designated values are specified or not. In our research on logics in which the designated values are specified, we are concerned ourselves with the validity of formulas if anything. On the other hand, we are obliged to deal with the validity of sequents introduced by Rousseau [6], Takahashi [7] and originally by Gentzen, in the study of those in which the designated values are not specified. Consequently, many-valued versions of Craig's interpolation theorem are also separated into two groups. Gill's version for a 3-valued logic in [1] and Rasiowa's for an $m$-valued logic $(m>2)$ in [5], not forgetting Craig's original theorem for the classical logic belong to the first group. In the meantime, examples of those of the second group are Miyama's version (see Fact 1 of this paper) in [4] and that (see Fact 2) by Hanazawa together with the present author in [2]; both followed Maehara who in [3] had given an elegant proof of Craig's theorem utilizing the cut-elimination theorem of Gentzen (see Takeuti [8]). As a by-product of syntactical proof of Rasiowa's, Wasilewska also obtained a version of the second group in [9].

In this paper, a (finitely) many-valued version of Craig's interpolation theorem of the first group will be investigated in a rather general setting. We remark that the choice of (the truth-value function of) "implication" is most problematic.

Suppose that a many-valued logic $L$ is given. Let $\boldsymbol{T}$ and $\boldsymbol{D}$ be the sets of truth-values and of designated values of $L$, respectively. We assume that $L$ is functionally complete in a strong sense, that is, for every function $f: T^{k} \rightarrow T$ there is a formula $f^{*}\left(P_{1}, \cdots, P_{k}\right)$ representing $f$ for each nonnegative integer $k$, and for every function $q:\{X \mid \varnothing \neq X \subseteq \boldsymbol{T}\} \rightarrow \boldsymbol{T}$ there is a formula $q^{*} x Q(x)$ representing $q$. Now we propose the following problem: Find the necessary and sufficient condition on $f: \boldsymbol{T}^{2} \rightarrow \boldsymbol{T}$ for which the interpolation property with respect to $f$ formulated below holds.

Received March 18, 1988 
INTERPOLATION PROPERTY WITH RESPECT TO $f$. If the truth-value of the formula $f^{*}(A, B)$ is always designated under any valuation, then there is a formula $C$ which satisfies the conditions (i) and (ii) below.

(i) The truth-values of $f^{*}(A, C)$ and $f^{*}(C, B)$ are always designated.

(ii) Every propositional letter, predicate letter and free individual variable occurring in $C$ occurs both in $A$ and $B$.

After preliminaries in $\S 1$, we shall prove the following theorem in $\S 2$ along the line of Rasiowa [5].

THEOREM. If $f: \boldsymbol{T}^{2} \rightarrow \boldsymbol{T}$ satisfies the conditions (a) and (b) below, then the interpolation property with respect to $f$ holds.

(a) If $f(\lambda, \mu) \in \boldsymbol{D}$ and $f(\mu, \nu) \in \boldsymbol{D}$, then $f(\lambda, \nu) \in \boldsymbol{D}$, for every $\lambda, \mu, \nu \in \boldsymbol{T}$.

(b) Either $f(\mu, \nu) \in \boldsymbol{D}$ or $f(\nu, \mu) \in \boldsymbol{D}$, for every $\mu, \nu \in \boldsymbol{T}$.

We shall show in $\S 3$ that Theorem covers Craig's original theorem, Gill's version in [1] and Rasiowa's in [5]. In the same section it will be remarked that, in Theorem we cannot replace the condition (b) by the weaker condition (b)' below.

(b) It is always the case that $f(\mu, \mu) \in \boldsymbol{D}$ for any $\mu \in \boldsymbol{T}$.

We shall also show in $\S 3$ that, the condition that (a) and (b) hold is not necessary for the interpolation property.

In [2], Hanazawa and the present author claimed that Miyama's version is an easy consequence of theirs. Afterwards the author noticed that, conversely the latter also follows easily from the former, which will be shown in $\S 4$ as an appendix.

The author expresses his hearty thanks to Prof. H. Rasiowa for her informing him of her article [5], without which he could hardly write this paper.

\section{$\S 1$. Preliminaries.}

Suppose that a (finitely) many-valued logic $L$ is given. To fix the idea, we assume that the sets $\boldsymbol{T}$ of truth-values and $\boldsymbol{D}$ of designated values of $L$ are the sets $\{\mu \mid 1 \leqq \mu \leqq M\}$ and $\{\mu \mid 1 \leqq \mu \leqq s\}$ respectively, where $M$ and $s$ are integers such that $1 \leqq s<M$. We also assume that for every function $f: \boldsymbol{T}^{k} \rightarrow \boldsymbol{T}$ there is a formula $f^{*}\left(P_{1}, \cdots, P_{k}\right)$ representing $f$ for each nonnegative integer $k$, and that for every function $q:\{X \mid \varnothing \neq X \subseteq \boldsymbol{T}\} \rightarrow \boldsymbol{T}$ there is a formula $q^{*} x Q(x)$ representing $q$.

An ordered pair of a formula and of a truth-value is called a valued formula. A sequent is a finite set of valued formulas. When $X \cong \boldsymbol{T}$, the sequent $\{(A, \mu) \mid \mu \in X\}$ is abbreviated as $(A, X)$. A singleton $\{(A, \mu)\}$ is written simply as $(A, \mu)$.

A sequent $K$ is valid if under any valuation, $K$ contains a valued formula $(A, \mu)$ such that $A$ takes the value $\mu$ under the valuation. Especially, the 
sequent $(A, \boldsymbol{D})$ is valid if and only if the truth value of $A$ is always designated under any valuation.

When $\Sigma$ is a formula or a sequent, $[\Sigma]$ denotes the set of propositional letters, predicate letters and free individual variables occurring in $\Sigma$.

Then, Miyama's interpolation theorem and that by Hanazawa together with the present author are stated as follows; the former will be utilized to the proof of Theorem.

FACT 1 (Miyama [4]). If $K \cup L$ is a valid sequent, then for every $\mu, \nu \in \boldsymbol{T}$ there is a formula $C$ such that $K \cup(C, \mu)$ and $L \cup(C, \nu)$ are valid and $[C] \cong$ $[K] \cap[L]$.

FACT 2 (Hanazawa and Takano [2]). If $K_{1} \cup \cdots \cup K_{M}$ is a valid sequent, then there is a formula $C$ such that $K_{\mu} \cup(C, \boldsymbol{T}-\{\mu\})$ is valid for every $\mu \in \boldsymbol{T}$ and $[C] \subseteq \cup\left\{\left[K_{\mu}\right] \cap\left[K_{\nu}\right] \mid 1 \leqq \mu<\nu \leqq M\right\}$.

\section{$\S 2$. Proof of Theorem.}

In view of proving Theorem, we suppose that the conditions (a) and (b) hold. We shall find a formula $C$ satisfying (i $)^{\prime}$ and (ii)' below under the assumption that the sequent $\left(f^{*}(A, B), \boldsymbol{D}\right)$ is valid.

(i) The sequents $\left(f^{*}(A, C), \boldsymbol{D}\right)$ and $\left(f^{*}(C, B), \boldsymbol{D}\right)$ are valid.

(ii) $^{\prime} \quad[C] \subseteq[A] \cap[B]$.

We call a formula $C$ an interpolant for $A$ and $B$, if (i) ${ }^{\prime}$ and (ii) ${ }^{\prime}$ are satisfied.

We define the binary relation $R$ on $\boldsymbol{T}$ by: $\mu R \nu$ if and only if $f(\mu, \nu) \in \boldsymbol{D}$. Then (a) and (b) mean that $R$ is transitive and connected (and hence reflexive), respectively. When $\mu \in X \subseteq T, \mu$ is minimal (maximal) in $X$ if $\mu R \nu(\nu R \mu$, resp.) for every $\nu \in X$. Clearly, every nonempty subset of $T$ has minimal elements and maximal ones.

If all the elements of $\boldsymbol{T}$ are minimal in $\boldsymbol{T}$, that is, $f(\mu, \nu) \in \boldsymbol{D}$ for every $\mu, \nu \in \boldsymbol{T}$, then the formula $\lambda^{*}$ for any $\lambda \in \boldsymbol{T}$ serves as an interpolant for arbitrary $A$ and $B$.

Consequently, we assume that not all the elements of $\boldsymbol{T}$ are minimal. Let $\sigma_{1}, \cdots, \sigma_{n}$ be the list of distinct elements of $\boldsymbol{T}$ which are not minimal $(1 \leqq n<M)$. For each $i=1,2, \cdots, n$, define the unary function $d_{\imath}$ on $\boldsymbol{T}$ by: $d_{i}(\mu)=1$ if $\sigma_{\imath} R \mu$, while $d_{i}(\mu)=M$ otherwise. Next, take an $n$-ary function $e$ on $\boldsymbol{T}$ such that: $e\left(\mu_{1}, \cdots, \mu_{n}\right)$ is one of the maximal elements of the set $\left\{\nu \in \boldsymbol{T} \mid \sigma_{k} R \nu\right.$ implies $\mu_{k}=1$, for every $\left.k=1,2, \cdots, n\right\}$ if it is nonempty, while $e\left(\mu_{1}, \cdots, \mu_{n}\right)$ is one of the minimal elements of $\boldsymbol{T}$ otherwise. Then we obtain the following proposition. 
PROPOSITION 1. $\left.1^{\circ}\right)$ For every $\mu, \nu \in T, \mu R \nu$ if and only if either $d_{i}(\mu)=M$ or $d_{i}(\nu)=1$ for every $i=1,2, \cdots, n$.

$\left.2^{\circ}\right)$ If $\sigma_{k} R \sigma_{\imath}$ and $d_{k}(\mu)=M$, then $d_{i}(\mu)=M$.

$\left.3^{\circ}\right)$ If $\sigma_{k} R \sigma_{i}$ implies $\mu_{k}=1$ for every $k=1,2, \cdots, n$, then $d_{i}\left(e\left(\mu_{1}, \cdots, \mu_{n}\right)\right)=1$.

$\left.4^{\circ}\right)$ If $\mu_{i}=M$, then $d_{i}\left(e\left(\mu_{1}, \cdots, \mu_{n}\right)\right)=M$.

Proof. $\left.1^{\circ}\right)$ Only-if-part. If $\mu R \nu$ but $d_{i}(\mu) \neq M$, then $d_{i}(\nu)=1$ by the transitivity of $R$. If-part. Assume that either $d_{i}(\mu)=M$ or $d_{i}(\nu)=1$ for every $i=1,2, \cdots, n$. If $\mu$ is minimal in $T$, then $\mu R \nu$ clearly. Suppose that $\mu$ is not minimal so that $\mu=\sigma_{2}$ for some $i$. Since $d_{i}(\mu)=1 \neq M$ by the reflexivity of $R, d_{i}(\nu)=1$ by the assumption, and so $\mu R \nu$ too.

$2^{\circ}$ ) Clear by the transitivity of $R$.

$\left.3^{\circ}\right)$ It follows from the hypothesis that $\sigma_{\imath} \operatorname{Re}\left(\mu_{1}, \cdots, \mu_{n}\right)$, and so $d_{i}\left(e\left(\mu_{1}, \cdots\right.\right.$, $\left.\left.\mu_{n}\right)\right)=1$.

$\left.4^{\circ}\right)$ Suppose $d_{i}\left(e\left(\mu_{1}, \cdots, \mu_{n}\right)\right) \neq M$. Then $\sigma_{\imath} \operatorname{Re}\left(\mu_{1}, \cdots, \mu_{n}\right)$. Since $\sigma_{2}$ is not minimal in $\boldsymbol{T}$, it follows by the transitivity of $R$ that $e\left(\mu_{1}, \cdots, \mu_{n}\right)$ is not minimal, either. Hence $\sigma_{k} \operatorname{Re}\left(\mu_{1}, \cdots, \mu_{n}\right)$ implies $\mu_{k}=1$, for every $k=1,2, \cdots, n$, and so $\mu_{i}=1 \neq M$.

Now, we are in the stage of proving Theorem. Suppose that the sequent $\left(f^{*}(A, B), \boldsymbol{D}\right)$ is valid. Then by Proposition $\left.1,1^{\circ}\right)$ it follows that $\left(d_{i}^{*}(A), M\right) \cup$ $\left(d_{2}^{*}(B), 1\right)$ is valid for every $i=1,2, \cdots, n$. So by Fact 1 there are formulas $C_{1}, C_{2}, \cdots, C_{n}$ such that for every $i=1,2, \cdots, n$, the following $(1 . i),(2 . i)$ and (3.i) hold.

The sequent $\left(d_{\imath}^{*}(A), M\right) \cup\left(C_{\imath}, 1\right)$ is valid.

The sequent $\left(d_{\imath}^{*}(B), 1\right) \cup\left(C_{\imath}, M\right)$ is valid.

$$
\left[C_{\imath}\right] \subseteq[A] \cap[B] .
$$

Then we claim that the formula $e^{*}\left(C_{1}, \cdots, C_{n}\right)$ serves as an interpolant for $A$ and $B$, that is, the following proposition holds.

Proposition 2. $\left.1^{\circ}\right)$ The sequent $\left(f^{*}\left(A, e^{*}\left(C_{1}, \cdots, C_{n}\right)\right), \boldsymbol{D}\right)$ is valid.

$\left.2^{\circ}\right)$ The sequent $\left(f^{*}\left(e^{*}\left(C_{1}, \cdots, C_{n}\right), B\right), \boldsymbol{D}\right)$ is valid.

$\left.3^{\circ}\right)\left[e^{*}\left(C_{1}, \cdots, C_{n}\right)\right] \subseteq[A] \cap[B]$.

Proof. $\left.1^{\circ}\right)$ Suppose $1 \leqq i \leqq n$. If $\sigma_{k} R \sigma_{\imath}$, then $\left(d_{i}^{*}(A), M\right) \cup\left(C_{k}, 1\right)$ is valid by $(1 . k)$ and Proposition $\left.1,2^{\circ}\right)$; hence $\left(d_{2}^{*}(A), M\right) \cup\left(d_{\imath}^{*}\left(e^{*}\left(C_{1}, \cdots, C_{n}\right)\right), 1\right)$ is valid by Proposition $\left.1,3^{\circ}\right)$. So we obtain $1^{\circ}$ ) by Proposition $\left.1,1^{\circ}\right)$.

$\left.2^{\circ}\right)$ From $(2 . i)$ and Proposition $\left.1,4^{\circ}\right)$ it follows that $\left(d_{i}^{*}(B), 1\right) \cup$ $\left(d_{i}^{*}\left(e^{*}\left(C_{1}, \cdots, C_{n}\right)\right), M\right)$ is valid, for every $i=1,2, \cdots, n$. Hence we obtain $\left.2^{\circ}\right)$ by Proposition $1,1^{\circ}$ ).

$3^{\circ}$ ) Clear by $(3.1), \cdots,(3 . n)$.

Thus we have completed the proof of Theorem. 


\section{§3. Scope of Theorem.}

In the classical logic (In the $m$-valued logic with which Rasiowa was concerned in [5]), $(M, s)=(2,1)((M, s)=(m, 1)$, resp.) and the truth-value function $f$ of the "implication" is defined by: $f(\mu, \nu)=1$ if $\mu \geqq \nu$, while $f(\mu, \nu)=\nu$ otherwise. Hence the conditions (a) and (b) are satisfied, and so Craig's original interpolation theorem (Rasiowa's version, resp.) is a special case of Theorem. On the other hand, in Gill's 3-valued logic investigated in $[1],(M, s)=(3,1)$ and the truth-value function $f$ of the "implication" is defined by: $f(\mu, \nu)=1$ if either $\mu \neq 1$ or $\nu=1$, while $f(\mu, \nu)=\nu$ otherwise. Hence Theorem covers Gill's version too, since (a) and (b) hold.

Next, we exemplify the fact that, in Theorem we cannot weaken the condition (b) into (b)'. Let $(M, s)=(4,1)$ and $f$ be a binary function on $\boldsymbol{T}=\{1,2,3,4\}$ such that: $f(\mu, \nu)=1$ if and only if either $\mu=\nu$ or $\mu>2 \geqq \nu$; then (a) and (b)' hold. Next, let $g$ and $h$ be the unary functions on $\boldsymbol{T}$ defined by: $g(\mu)=3$ and $h(\mu)=1$ if $\mu \leqq 2$, while $g(\mu)=4$ and $h(\mu)=2$ otherwise. Then $\left(f^{*}\left(g^{*}(P), h^{*}(Q)\right), 1\right)$ is valid, where $P$ and $Q$ are distinct propositional letters. But there is no interpolant for $g^{*}(P)$ and $h^{*}(Q)$, since none of $\left(f^{*}\left(1^{*}, h^{*}(Q)\right), 1\right)$, $\left(f^{*}\left(2^{*}, h^{*}(Q)\right), 1\right),\left(f^{*}\left(g^{*}(P), 3^{*}\right), 1\right)$ and $\left(f^{*}\left(g^{*}(P), 4^{*}\right), 1\right)$ are valid. Hence the interpolation property with respect to $f$ does not hold.

We finally remark that the condition that (a) and (b) hold is not necessary for the interpolation property. To show this, let $(M, s)=(3,1)$ and $f$ be a binary function on $\boldsymbol{T}=\{1,2,3\}$ such that: $f(\mu, \nu)=1$ if and only if either $\mu=3$ or $\nu=1$; then (a) is satisfied but (b) is not. With a view to showing the interpolation property, suppose that $\left(f^{*}(A, B), 1\right)$ is valid. Then $(A, 3) \cup(B, 1)$ is valid, and so $(A, 3) \cup(C, 1)$ and $(B, 1) \cup(C, 3)$ are valid and $[C] \subseteq[A] \cap[B]$ for some $C$ by Fact 1 . Hence $C$ serves as an interpolant for $A$ and $B$, since $\left(f^{*}(A, C), 1\right)$ and $\left(f^{*}(C, B), 1\right)$ are valid.

\section{§4. Appendix.}

In this section, we shall derive Fact 2 from Fact 1 . For this purpose, we assume that Fact 1 holds and

$$
K_{1} \cup \cdots \cup K_{M} \text { is valid. }
$$

From (4) by Fact 1 it follows that, there is a formula $C_{1}$ such that (5.1), (6.1) and (7.1) below hold.

$$
\begin{gathered}
K_{1} \cup\left(C_{1}, 1\right) \text { is valid. } \\
K_{2} \cup \cdots \cup K_{M} \cup\left(C_{1}, M\right) \quad \text { is valid. } \\
{\left[C_{1}\right] \subseteq\left[K_{1}\right] \cap\left[K_{2} \cup \cdots \cup K_{M}\right] .}
\end{gathered}
$$


From (6.1) by Fact 1 it follows that, there is a formula $C_{2}$ such that (5.2), (6.2) and (7.2) below hold.

$$
\begin{gathered}
K_{2} \cup\left(C_{2}, 1\right) \quad \text { is valid. } \\
K_{3} \cup \cdots \cup K_{M} \cup\left(C_{1}, M\right) \cup\left(C_{2}, M\right) \text { is valid. } \\
{\left[C_{2}\right] \subseteq\left[K_{2}\right] \cap\left[K_{3} \cup \cdots \cup K_{M} \cup\left(C_{1}, M\right)\right] .}
\end{gathered}
$$

From (6.2) by Fact 1 again it follows that, there is a formula $C_{3}$ such that (5.3), (6.3) and (7.3) below hold.

$$
K_{3} \cup\left(C_{3}, 1\right) \text { is valid. }
$$

$$
K_{4} \cup \cdots \cup K_{M} \cup\left(C_{1}, M\right) \cup\left(C_{2}, M\right) \cup\left(C_{3}, M\right) \text { is valid. }
$$

$$
\left[C_{3}\right] \subseteq\left[K_{3}\right] \cap\left[K_{4} \cup \cdots \cup K_{M} \cup\left(C_{1}, M\right) \cup\left(C_{2}, M\right)\right] .
$$

Continuing in this way, we finally obtain a formula $C_{M-1}$ such that $(5 . M-1)$, $(6 . M-1)$ and $(7 . M-1)$ below hold.

$$
K_{M-1} \cup\left(C_{M-1}, 1\right) \text { is valid. }
$$

$$
K_{M} \cup\left(C_{1}, M\right) \cup \cdots \cup\left(C_{M-2}, M\right) \cup\left(C_{M-1}, M\right) \text { is valid. }
$$$$
\left[C_{M-1}\right] \subseteq\left[K_{M-1}\right] \cap\left[K_{M} \cup\left(C_{1}, M\right) \cup \cdots \cup\left(C_{M-2}, M\right)\right] \text {. }
$$

Next, take an $(M-1)$-ary function $g$ on $\boldsymbol{T}$ such that: $g\left(\mu_{1}, \cdots, \mu_{M-1}\right)$ is one of such $i$ for which $\mu_{i} \neq 1$ if any, while $g\left(\mu_{1}, \cdots, \mu_{M-1}\right)=M$ otherwise. Then clearly we have (8) and (9) below.

(8) For every $i=1,2, \cdots, M-1$, if $\mu_{2}=1$, then $g\left(\mu_{1}, \cdots, \mu_{M-1}\right) \neq i$.

(9) If either $\mu_{1}=M, \cdots, \mu_{M-2}=M$ or $\mu_{M-1}=M$, then $g\left(\mu_{1}, \cdots, \mu_{M-1}\right) \neq M$.

Now, we claim that $g^{*}\left(C_{1}, \cdots, C_{M-1}\right)$ is the desired formula, that is, the following proposition hold.

Proposition 3. $\left.1^{\circ}\right)$ The sequent $K_{\mu} \cup\left(g^{*}\left(C_{1}, \cdots, C_{M-1}\right), \boldsymbol{T}-\{\mu\}\right)$ is valid for every $\boldsymbol{\mu} \in \boldsymbol{T}$.

$\left.2^{\circ}\right) \quad\left[g^{*}\left(C_{1}, \cdots, C_{M-1}\right)\right] \leqq \cup\left\{\left[K_{\mu}\right] \cap\left[K_{\nu}\right] \mid 1 \leqq \mu<\nu \leqq M\right\}$.

Proof. $1^{\circ}$ ) We have $1^{\circ}$ ) by $(6 . M-1)$ with (9) or $(5 . \mu)$ with (8) according as $\mu=M$ or not.

$2^{\circ}$ ) It suffices to prove for every $i=1,2, \cdots, M-1$,

$$
\left[C_{\imath}\right] \subseteq\left[K_{\imath}\right] \cap\left[K_{\imath+1} \cup \cdots \cup K_{M} \cup K_{1} \cup \cdots \cup K_{\imath-1}\right],
$$

which we prove by induction on $i$. From the hypothesis of induction it follows that $\left[C_{1}\right] \subseteq\left[K_{1}\right], \cdots,\left[C_{\imath-1}\right] \subseteq\left[K_{\imath-1}\right]$, and so we obtain (10) by $(7 . i)$. 
Thus we have deduced Fact 2 from Fact 1.

\section{REFERENCES}

[1] R. R. R. GILL, The Craig-Lyndon interpolation theorem in 3-valued logic, J. Symbolic Logic, 35 (1970), 230-238.

[2] M. HANAZAWA AND M. TAKANO, An interpolation theorem in many-valued logic, J. Symbolic Logic, 51 (1986), 448-452.

[3] S. MAEhARA, On the interpolation theorem of Craig (in Japanese), Sûgaku, 12 (1960), 235-237.

[4] T. Miyama, The interpolation theorem and Beth's theorem in many-valued logics, Math. Japan., 19 (1974), 341-355.

[5] H. RAsiowa, The Craig interpolation theorem for $m$-valued predicate calculi, Bull. Acad. Polon. Sci., Sér. Sci. Math. Astronom. Phys., 20 (1972), 341-346.

[6] G. Rousseau, Sequents in many valued logic I, Fund. Math., 60 (1967), 23-33.

[7]. M. Takahashi, Many-valued logics of extended Gentzen style I, Sci. Rep. Tokyo Kyoiku Daigaku, Sect. A, 9 (1967), 271-292.

[8] G. Takeuti, Proof Theory, North-Holland, Amsterdam, 1975.

[9] A. WAsilewska, A constructive proof of Craig's interpolation lemma for $m$-valued logic, Studia Logica, 38 (1979), 267-275.

Department of Mathematics

FACULTY OF EDUCATiON

Nigata University

Nigata, 950-21, JAPAN 